\title{
A nationwide survey on non-B, non-C hepatocellular carcinoma in Japan: 2011-2015 update
}

\author{
Ryosuke Tateishi $^{1}$ (1) Koji Uchino $^{1} \cdot$ Naoto Fujiwara $^{1} \cdot$ Tetsuo Takehara $^{2} \cdot$ \\ Takeshi Okanoue $^{3} \cdot$ Masataka Seike $^{4} \cdot$ Hitoshi Yoshiji $^{5} \cdot$ Hiroshi Yatsuhashi $^{6}$. \\ Masahito Shimizu ${ }^{7} \cdot$ Takuji Torimura $^{8}$ - Mitsuhiko Moriyama9 ${ }^{9}$ Isao Sakaida ${ }^{10}$. \\ Hiroyuki Okada ${ }^{11}$ - Tetsuhiro Chiba ${ }^{12}$ - Makoto Chuma ${ }^{13} \cdot$ Kazuhiko Nakao $^{14}$. \\ Hajime Isomoto $^{15} \cdot$ Yutaka Sasaki $^{16}$ - Shuichi Kaneko ${ }^{17}$ • Tsutomu Masaki ${ }^{18}$. \\ Kazuaki Chayama $^{19} \cdot$ Kazuhiko Koike $^{1}$
}

Received: 1 October 2018/Accepted: 21 November 2018/Published online: 29 November 2018

(C) The Author(s) 2018

\begin{abstract}
Background We previously reported that the incidence of hepatocellular carcinoma (HCC) with non-viral etiologies increased rapidly between 1991 and 2010 in Japan.

Methods To update this investigation, we enrolled patients who were initially diagnosed as having non-B, non-C HCC at participating hospitals between 2011 and 2015. In addition to the patient characteristics investigated in the previous report, we also investigated the duration of
\end{abstract}

Electronic supplementary material The online version of this article (https://doi.org/10.1007/s00535-018-1532-5) contains supplementary material, which is available to authorized users.

Ryosuke Tateishi

tateishi-tky@umin.ac.jp

1 Department of Gastroenterology, Graduate School of Medicine, The University of Tokyo, 7-3-1 Hongo, Bunkyo$\mathrm{Ku}$, Tokyo 113-8655, Japan

2 Department of Gastroenterology and Hepatology, Osaka University Graduate School of Medicine, Suita, Japan

3 Department of Gastroenterology and Hepatology, Saiseikai Suita Hospital, Suita, Japan

4 Depatment of Gastroenterology, Faculty of Medicine, Oita University, Yufu, Japan

5 Third Department of Internal Medicine, Nara Medical University, Kashihara, Japan

6 Clinical Research Center, National Hospital Organization (NHO) Nagasaki Medical Center, Nagasaki University Graduate School of Biomedical Sciences, Nagasaki, Japan

7 Department of Gastroenterology, Gifu University Graduate School of Medicine, Gifu, Japan

8 Division of Gastroenterology, Department of Internal Medicine, Kurume University School of Medicine, Kurume, Japan alcohol consumption. The overall survival rate was analyzed using the Kaplan-Meier method, and the hazard function against the body mass index (BMI) was plotted using cubic splines.

Results A total of 2087 patients were enrolled. The proportion of patients with non-viral etiologies has continued to increase from $10.0 \%$ in 1991 to $32.5 \%$ in 2015 . Patients were also older (median ages, 70-73 years) and more obese (median BMIs, $23.9-24.2 \mathrm{~kg} / \mathrm{m}^{2}$ ), and the proportions of patients with diabetes mellitus ( $46.1 \%$ to $51.6 \%$ ), hypertension ( $42.7 \%$ to $58.6 \%$ ), dyslipidemia (14.6\% to $22.9 \%$ ), and fatty liver (24.0\% to $28.8 \%$ ) had all increased

9 Division of Gastroenterology and Hepatology, Department of Internal Medicine, Nihon University School of Medicine, Tokyo, Japan

10 Department of Gastroenterology and Hepatology, Yamaguchi University Graduate School of Medicine, Ube, Japan

11 Department of Gastroenterology and Hepatology, Okayama University Graduate School of Medicine, Dentistry and Pharmaceutical Sciences, Okayama, Japan

12 Department of Gastroenterology and Nephrology, Graduate School of Medicine, Chiba University, Chiba, Japan

13 Gastroenterological Center, Yokohama City University Medical Center, Yokohama, Japan

14 Department of Gastroenterology and Hepatology, Nagasaki University of Graduate School of Biomedical Sciences, Nagasaki, Japan

15 Faculty of Medicine, Medicine and Clinical Science, Tottori University, Yonago, Japan

16 Department of Gastroenterology and Hepatology, Graduate School of Medical Sciences, Kumamoto University, Kumamoto, Japan

17 Department of Gastroenterology, Graduate School of Medicine, Kanazawa University, Kanazawa, Japan 
significantly. There was a significant inverse relationship between the duration and the amount of daily alcohol consumption. The improvement in the overall survival was relatively small, with a decreased proportion of patients under surveillance $(41.3 \%$ to $31.6 \%)$. A hazard function plot showed a curve similar to that in our previous report, with a lowest hazard of $\sim 26 \mathrm{~kg} / \mathrm{m}^{2}$.

Conclusions The proportion of HCC patients with nonviral etiologies continues to increase in Japan. Lifetime total amount of alcohol consumption may be a risk factor.

Keywords Hepatocellular carcinoma $\cdot$ Non-alcoholic fatty liver disease $\cdot$ Alcoholic liver disease

\section{Introduction}

Chronic hepatitis $\mathrm{B}$ and $\mathrm{C}$ has contributed to primary liver cancer, especially the development of hepatocellular carcinoma (HCC), in the vast majority of such cases worldwide [1]. As a result of efforts to control hepatitis B (HBV) and hepatitis $\mathrm{C}$ virus (HCV) infections, mortality rates have started to decrease in Asia and Southern Europe [2]. On the other hand, the mortality rates continue to increase in other regions such as the United States, where the increase in the incidence of HCC was the highest among all types of cancer between 2003 and 2012 [3].

Growing evidence suggests that obesity and diabetes increase various cancer risks [4-8], with obesity and obesity-associated conditions having the largest impact on carcinogenic processes in the liver $[5,6,9,10]$. In fact, the population-attributable fractions of obesity and diabetes on liver cancer development were the largest among various risk factors in the United States, suggesting that the diversity in obese populations among races and ethnicities has contributed to the disparity in the increasing rates of HCC in the United States [11].

We previously reported that the incidence of HCC with a non-viral etiology increased rapidly between 1991 and 2010 and that the proportion of patients with obesity or diabetes was higher than that of patients with viral hepatitis during this period. We concluded that the increase in the obese population among Japanese males over the last three decades has contributed to the increase in HCC patients with a non-viral etiology [12]. The present study aims to update this investigation by collecting data from 2011 to 2015 and by including additional information regarding

18 Department of Gastroenterology and Neurology, Faculty of Medicine, Kagawa University, Takamatsu, Japan

19 Department of Gastroenterology and Metabolism, Graduate School of Biomedical and Health Sciences, Hiroshima University, Hiroshima, Japan detailed histories of alcohol consumption and prescriptions for diabetes, hypertension, and dyslipidemia.

\section{Patients and methods}

\section{Study design}

This retrospective study complied with the ethical guidelines for medical and health research involving human subjects established by the Japanese Ministry of Education, Culture, Sports, Science, and Technology and the Ministry of Health, Labour, and Welfare. The study protocol was approved by the University of Tokyo Medical Research Center Ethics Committee (approval number 3710) and the Institutional Review Board or Ethics Committee of each participating institution. Informed consent was waived because of the retrospective design. This study was registered with the University Hospital Medical Information Network (UMIN) Clinical Trial Registry (UMINCTR000007570).

\section{Patients}

In the current study, we collected data from patients who were initially diagnosed as having non-B, non-C $\mathrm{HCC}$ at the participating hospitals between 2011 and 2015. Patients who were negative for both hepatitis B surface antigen (HBsAg) and anti-HCV antibody were enrolled. We also investigated the number of HCC patients who were initially diagnosed during the same period according to the following four categories: HBV single positive, $\mathrm{HCV}$ single positive, both $\mathrm{HBV}$ and $\mathrm{HCV}$ positive, and non-B, non-C. We also used data from our previous study, which had enrolled non-B, non-C HCC patients diagnosed between 1991 and 2010, for comparison purposes [12].

\section{Diagnosis of HCC}

HCC was diagnosed pathologically or using imaging criteria based on the Japanese Clinical Practice Guidelines; hyperattenuation during the arterial phase with washout during the late phase on dynamic CT or dynamic MRI images was regarded as a specific finding [13].

\section{Data collection}

The patients were registered via an electronic data capture system designed by the investigators. We collected data at the time of the initial diagnosis of HCC; this data included anthropometric parameters, daily alcohol consumption, medical comorbidities, tumor characteristics, treatment modalities and laboratory data. The collected items were 
described in detail in our previous report [12]. The body mass index (BMI), Child-Turcotte-Pugh (CTP) score [14], fibrosis-4 (FIB-4) index [15], and Barcelona Clinic Liver Cancer (BCLC) stage [16] were calculated automatically using the obtained data.

The etiologies of the background liver diseases were categorized as follows: autoimmune hepatitis (AIH), primary biliary cholangitis (PBC), AIH-PBC overlap syndrome, alcoholic liver disease (ALD), non-alcoholic fatty liver disease (NAFLD), Budd-Chiari syndrome, hemochromatosis, Wilson's disease, and others. Diagnoses of background liver diseases, diabetes mellitus, hypertension, and dyslipidemia were made by the attending physician based on the Japanese clinical guidelines for each disease. Daily alcohol consumption was calculated from reported forms regarding alcohol intake and frequency. Regarding the diagnosis of alcoholic liver disease, we used previously reported criteria of a daily alcohol consumption $\geq 80 \mathrm{~g} /$ day and the absence of any other definite etiology of liver disease in our previous report. In the present report, however, we revised the criterion for alcohol consumption to $\geq 60 \mathrm{~g} /$ day based on the Japanese Society for Biomedical Research on Alcohol (JASBRA) Diagnostic Criteria for Alcoholic Liver Disease 2011 Edition [17]. The background etiologies in the 1991-2010 survey were recalculated based on this new criterion. NAFLD was defined as the presence or a history of fatty liver diagnosed radiologically or pathologically and an alcohol consumption of $\leq 20 \mathrm{~g} /$ day.

The survival statuses of the patients were also registered. Survival status was defined as alive, dead, or lost to follow-up. Observations were censored as of December 31, 2015. For the deceased patients, the cause of death was categorized according to the criteria of the Liver Cancer Study Group of Japan [18] as follows: liver cancer progression, liver failure, gastrointestinal bleeding, gastroesophageal varices rupture, rupture of liver cancer, operative death, other, or unknown.

In addition to the data investigated in our previous report, we also collected the following data in the present study. First, we investigated the history of fatty liver. Second, we investigated the ages at which the patients started and stopped drinking and whether the patients had practiced moderation in their drinking for at least 6 months prior to the diagnosis of HCC. Moderation in drinking was defined as decreased amount of daily alcohol consumption from the peak value. We also compared the $\gamma$-glutamyltransferase (GGT), aspartate aminotransferase (AST), and alanine aminotransferase (ALT) values and the FIB-4 index between patients who had practiced moderation in their drinking for longer than 6 months prior to the diagnosis of HCC and patients who had continued to drink, matched for gender and daily amount of alcohol intake.
Finally, we investigated the presence of prescriptions for the treatment of diabetes, hypertension, and dyslipidemia.

\section{Statistical analysis}

Data are expressed as the medians with the 25 th to 75 th percentiles unless otherwise indicated. Numbers and percentages were used for qualitative variables. The Student's $t$ test or the Wilcoxon rank-sum test was used for comparisons between two continuous variables. For the comparison of paired data, the Wilcoxon signed-rank test was used. Differences among groups were assessed using a oneway analysis of variance (ANOVA) for continuous data and the Chi-squared test for categorical data. The CochranArmitage trend test was used to evaluate increasing or decreasing trends. Survival time was defined as the interval between the day of the initial diagnosis and death or the last visit to the hospital. Cumulative survival curves were constructed using the Kaplan-Meier method and were compared using the log-rank test. To assess the hazard ratios of various factors on overall survival, the Cox proportional hazard model was used. We also plotted the relative hazard against BMI using cubic splines.

All the statistical analyses were performed using $\mathrm{R}$ software (ver. 3.2.3; R Development Core Team, Vienna, Austria). All the tests were two-sided, and $P$ values $<0.05$ were considered to indicate statistical significance.

\section{Results}

\section{Patient profiles}

Thirty-four hospitals consented to participate in the current survey. Of the 7,370 patients who were initially diagnosed as having $\mathrm{HCC}$ at the participating hospitals, 2087 (28.3\%) were categorized as non-B, non-C. The proportion of patients with a non-B, non-C etiology continued to increase in 2011-2015 (Fig. 1; $P<0.001$, Cochran-Armitage test). The proportion of non-B, non-C patients increased from $10.0 \%$ in 1991 to $32.5 \%$ in 2015 . The distribution of background liver diseases among non-B, non-C patients was as follows: AIH in 54 (2.6\%), PBC in 52 (2.5\%), AIHPBC overlap syndrome in $10(0.5 \%)$, alcoholic liver disease in 675 (32.3\%), NAFLD in 315 (15.1\%), Budd-Chiari Syndrome in $1(0.04 \%)$, hemochromatosis in $2(0.1 \%)$, Wilson's disease in $4(0.2 \%)$, unclassified in 911 (43.7\%), and other in $63(3.0 \%)$.

We compared the baseline characteristics of the current study cohort with the previous cohort (Table 1). The age at the time of diagnosis increased significantly, whereas the proportion of males was almost the same. The BMI increased slightly, whereas the proportions of patients with 


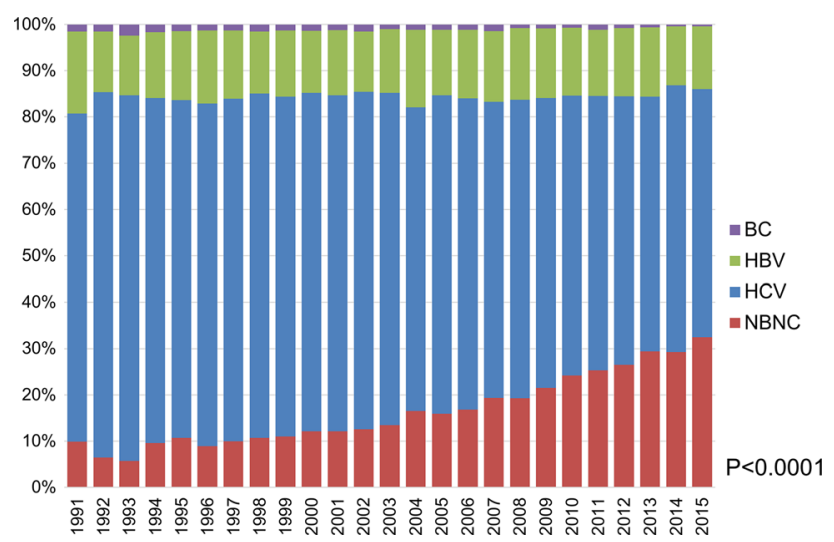

Fig. 1 Trend in background liver diseases among patients with hepatocellular carcinoma in Japan. The current and previous cohorts were combined. A marked increase in the proportion of patients categorized as non-B, non-C was observed at the participating hospitals $(P<0.0001$, Cochran-Armitage test $)$

diabetes mellitus, hypertension, dyslipidemia, and fatty liver increased significantly. Although HCC was diagnosed at a slightly earlier stage in the current cohort, the difference was quite small.

Regarding the HCC diagnostic process, liver nodules were initially pointed out in $18.5 \%$ of the patients at the participating departments, and $31.6 \%$ were followed with imaging modalities before diagnosis. The proportion of patients under surveillance decreased, compared with that in the previous study (41.3\%).

\section{Fatty liver and detailed history of alcohol consumption}

Fatty liver was observed in 502 patients $(28.8 \%)$ at the time of the diagnosis of HCC. Of the 1,656 patients in whom a previous history of fatty liver disease had been documented, fatty liver was observed in $449(27.1 \%)$ prior to the diagnosis of HCC. Of these patients, 417 (92.9\%) still exhibited fatty liver at the time of the diagnosis of HCC, whereas the fatty liver had disappeared in $32(7.1 \%)$.

Regarding alcohol consumption, 1503 (72.0\%) patients were former or current drinkers. Of them, the duration of drinking was available in 890. A significant inverse relationship between the duration of drinking and the amount of daily alcohol consumption was observed (Fig. 2, $P<0.0001$, Cochran-Armitage test). The presence or absence of moderation in drinking prior to the diagnosis of HCC was known for 1,181 of the 1,503 patients. A total of 414 patients had practiced moderation in drinking, and we selected another group of patients without moderation in drinking by matching the groups for sex and daily amount of alcohol consumption in a 1:1 ratio. Consequently, 311 patients were retrieved in each group. The baseline characteristics of the patients are shown in Table 2. The differences in values between the two groups were most prominent for GGT, followed by AST; the difference in the ALT values was much smaller, and no significant difference in the FIB-4 indices was seen (Supplementary Fig. 1).

\section{Medication for diabetes, hypertension, and dyslipidemia}

Regarding medications for diabetes, dipeptidyl peptidase-4 inhibitors were the most common, followed by sulfonylurea. The proportion of patients using insulin was $23.3 \%$. Calcium channel blockers were the most common antihypertensive medications, followed by angiotensin II receptor blockers. Statins were used in the vast majority of patients with dyslipidemia (Supplementary Fig. 2).

\section{Treatment and survival}

The distribution of initial treatments was as follows: resection in $452(21.7 \%)$, ablation in $394(18.9 \%)$, ablation + TACE in 187 (9.0\%), TACE in 656 (31.4\%), transarterial chemotherapy with one-shot and continuous infusion in $146(7.0 \%)$, systemic therapy in $58(2.8 \%)$, radiation therapy in $24(1.1 \%)$, liver transplantation in $8(0.4 \%)$, others in $33(1.6 \%)$, and supportive care in $129(6.2 \%)$. The distribution was quite similar to the previous report.

During the mean follow-up period of 2.1 years, 704 patients died and 272 patients were lost to follow-up. The causes of death were cancer progression in 465 (66.1\%), liver failure in $63(8.9 \%)$, gastrointestinal bleeding (including varices rupture) in $22(3.1 \%)$, tumor rupture in 18 (2.6\%), and others in $92(13.1 \%)$. The cause of death was unspecified in $44(6.3 \%)$. The median survival time $(95 \%$ confidence interval $[\mathrm{CI}]$ ) after the initial diagnosis of HCC was $4.52(4.05-5.11)$ years. The overall survival rates at 1 , 2, 3, and 5 years were 83.9, 72.6, 61.9, and 46.4\%, respectively (Fig. 3). The survival was slightly improved, compared with the previous report $(P<0.0001$, log-rank test). The hazard ratio of the new cohort versus the previous cohort was 0.898 (95\% CI, 0.819-0.985) in the multivariate analysis using age, gender, and tumor- and liver function-related factors (Table 3). The estimated hazard plot according to the BMI exhibited J-shaped curves in both the current and previous cohorts (Fig. 4). The shape of the curves was quite similar to the previous report, with the lowest hazard at around $26 \mathrm{~kg} / \mathrm{m}^{2}$.

\section{Discussion}

In the present study, we confirmed that the proportion of patients with a non-viral etiology continues to increase in Japan, as reported in our previous study. The numbers of 
Table 1 Baseline characteristics of HCC patients

\begin{tabular}{|c|c|c|c|}
\hline & 2011-2015 & 1991-2010 & $P$ value \\
\hline Number of patients & 2087 & 5326 & \\
\hline Etiology & & & $<0.0001$ \\
\hline AIH & $54(2.6)$ & $161(3.0)$ & \\
\hline $\mathrm{PBC}$ & $52(2.5)$ & $166(3.1)$ & \\
\hline Alcoholic liver disease & $675(32.3)$ & $1997(37.5)$ & \\
\hline NAFLD & $315(15.1)$ & $596(11.2)$ & \\
\hline Unclassified & $911(43.7)$ & $2301(43.2)$ & \\
\hline Others & $80(3.8)$ & $105(2.0)$ & \\
\hline Age, year & $73.0(66.0-78.0)$ & $70.0(63.0-75.0)$ & $<0.0001$ \\
\hline Male gender, $n(\%)$ & $1560(74.7)$ & $4022(75.5)$ & 0.51 \\
\hline BMI $\left(\mathrm{kg} / \mathrm{m}^{2}\right)$ & $24.2(21.7-27.0)$ & $23.9(21.6-26.6)$ & 0.003 \\
\hline Alcohol consumption $(\mathrm{g} / \text { day })^{\mathrm{a}}$ & & & 0.53 \\
\hline$\leq 20, n(\%)$ & $1058(50.7)$ & $2623(50.9)$ & \\
\hline $21-59, n(\%)$ & $263(12.6)$ & $602(11.7)$ & \\
\hline$\geq 60, n(\%)$ & $766(36.7)$ & $1928(37.4)$ & \\
\hline Diabetes, $n(\%)^{\mathrm{b}}$ & $1072(51.6)$ & $2345(46.1)$ & $<0.0001$ \\
\hline Hypertension, $n(\%)^{\mathrm{c}}$ & $1201(58.6)$ & $2063(42.7)$ & $<0.0001$ \\
\hline Dyslipidemia, $n(\%)^{\mathrm{d}}$ & $448(22.9)$ & $720(14.6)$ & $<0.0001$ \\
\hline Fatty liver, $n(\%)^{\mathrm{e}}$ & $502(28.8)$ & $936(24.0)$ & $<0.0001$ \\
\hline Anti-HBc Ab positive, $n(\%)^{\mathrm{f}}$ & $618(35.3)$ & $1501(40.3)$ & $<0.0001$ \\
\hline ALT (U/L) & $30(21-46)$ & $32(22-50)$ & $<0.0001$ \\
\hline Platelet count $\left(\times 10^{9} / \mu \mathrm{L}\right)^{\mathrm{g}}$ & $14.0(9.70-19.50)$ & $135(90-193)$ & 0.002 \\
\hline FIB-4 index ${ }^{\mathrm{h}}$ & $4.15(2.56-6.50)$ & $4.06(2.50-6.71)$ & 0.96 \\
\hline Child-Pugh class ${ }^{\mathrm{i}}$ & & & 0.005 \\
\hline $\mathrm{A}, n(\%)$ & $1445(69.7)$ & $3500(69.0)$ & \\
\hline $\mathrm{B}, n(\%)$ & $530(25.6)$ & $1231(24.3)$ & \\
\hline $\mathrm{C}, n(\%)$ & $97(4.7)$ & $338(6.7)$ & \\
\hline Tumor characteristics & & & 0.49 \\
\hline Maximal tumor size $(\mathrm{cm})^{\mathrm{j}}$ & $3.1(2.0-6.2)$ & $3.2(2.0-6.0)$ & \\
\hline Number of nodules ${ }^{\mathrm{k}}$ & & & 0.019 \\
\hline Single, $n(\%)$ & $1139(54.6)$ & $2700(51.1)$ & \\
\hline $2-3, n(\%)$ & $488(23.4)$ & $1368(25.9)$ & \\
\hline$>3, n(\%)$ & $460(22.0)$ & $1220(23.1)$ & \\
\hline Vascular invasion $^{1}, n(\%)$ & $53(2.5)$ & $187(3.5)$ & 0.036 \\
\hline Extrahepatic metastasis $^{\mathrm{m}}, n(\%)$ & $189(9.1)$ & $401(7.6)$ & 0.038 \\
\hline \multicolumn{4}{|l|}{$\mathrm{AFP}^{\mathrm{n}}(\mathrm{ng} / \mathrm{mL})$} \\
\hline$\leq 20, n(\%)$ & $1271(62.0)$ & $2908(59.4)$ & 0.016 \\
\hline $21-200, n(\%)$ & $357(17.4)$ & $820(16.8)$ & \\
\hline$>200, n(\%)$ & $423(20.6)$ & $1164(23.8)$ & \\
\hline \multicolumn{4}{|l|}{$\mathrm{DCP}^{\mathrm{o}}(\mathrm{mAU} / \mathrm{mL})$} \\
\hline$\leq 100, n(\%)$ & $949(47.0)$ & $2121(45.8)$ & \\
\hline $101-400, n(\%)$ & $308(37.8)$ & 787 (17.0) & \\
\hline$>400, n(\%)$ & $764(37.8)$ & $1727(37.3)$ & \\
\hline $\operatorname{AFP}^{-L} 3^{\mathrm{P}}(\%)$ & & & $<0.0001$ \\
\hline$\leq 10, n(\%)$ & $832(59.2)$ & $1765(67.7)$ & \\
\hline $10.1-15, n(\%)$ & 99 (7.0) & $74(2.8)$ & \\
\hline$>15, n(\%)$ & $475(33.8)$ & 767 (29.4) & \\
\hline BCLC stage ${ }^{\mathrm{q}}$ & & & $<0.0001$ \\
\hline $0 / \mathrm{A}, n(\%)$ & $1083(51.9)$ & 26n (49.6) & \\
\hline $\mathrm{B}, n(\%)$ & 703 (33.7) & $2023(38.3)$ & \\
\hline
\end{tabular}


Table 1 continued

\begin{tabular}{lcll}
\hline & $2011-2015$ & $1991-2010$ & $P$ value \\
\hline $\mathrm{C}, n(\%)$ & $207(9.9)$ & $312(5.9)$ & \\
$\mathrm{D}, n(\%)$ & $94(4.5)$ & $329(6.2)$ &
\end{tabular}

Data are expressed as the median (25th-75th percentiles) or number (percentages)

$A F P$ alpha-fetoprotein, AFP-L3 Lens culinaris agglutinin-reactive fraction of AFP, AIH autoimmune hepatitis, $A L T$ alanine aminotransferase, Anti-HBc Ab anti-hepatitis B core antibody, BCLC Barcelona Clinic Liver Cancer, $B M I$ body mass index, $D C P$ des-gamma-carboxy prothrombin, FIB-4 fibrosis-4

Since only a few patients were categorized as having AIH-PBC overlap syndrome, Budd-Chiari syndrome, hemochromatosis and Wilson's disease, these categories were combined with 'others'. Data were missing for ${ }^{\mathrm{a}} 173,{ }^{\mathrm{b}} 241,{ }^{\mathrm{c}} 498,{ }^{\mathrm{d}} 388,{ }^{\mathrm{e}} 1434,{ }^{\mathrm{f}} 1606,{ }^{\mathrm{g}} 61,{ }^{\mathrm{h}} 142,{ }^{\mathrm{i}} 257,{ }^{\mathrm{j}} 42,{ }^{\mathrm{k}} 38,{ }^{\mathrm{l}} 28,{ }^{\mathrm{m}} 26,{ }^{\mathrm{n}} 434,{ }^{\mathrm{o}} 691,{ }^{\mathrm{p}} 3677$, and ${ }^{\mathrm{q}} 40$ patients in the 1991-2010 cohort and ${ }^{\mathrm{b}} 10,{ }^{\mathrm{c}} 36,{ }^{\mathrm{d}} 128,{ }^{\mathrm{e}} 346,{ }^{\mathrm{g}} 312,{ }^{\mathrm{i}} 15,{ }^{\mathrm{n}} 36,{ }^{\mathrm{o}} 66$, and ${ }^{\mathrm{p}} 681$ patients in the 2011-2015 cohort, respectively

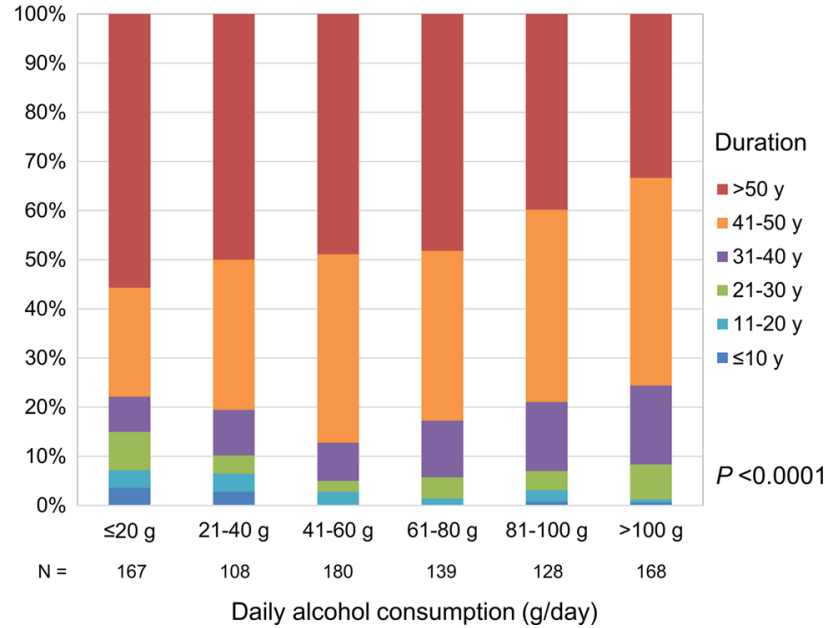

Fig. 2 Duration and daily amount of alcohol consumption. Those who consumed a larger amount of alcohol daily tended to require a shorter duration of drinking to develop hepatocellular carcinoma $(P<0.0001$, Cochran-Armitage test $)$

patients with diabetes mellitus, hypertension, dyslipidemia, and fatty liver had all increased, compared with the values in our previous study, whereas alcoholic consumption was similar. The increase in the proportion of non-B, non-C
HCC can be partially explained by a decrease in the number of HCV-related HCC cases, since the number of HCV carriers in Japan has continued to decrease. However, when the number of patients with a non-viral etiology was calculated by multiplying the proportion observed in the present study and the annual incidences of liver cancer reported by the Japanese government (data not shown), an ongoing increase was observed [19].

On the other hand, tumor characteristics and liver function did not change between the two studies. Although HCC seems to have been diagnosed at a slightly earlier stage, based on the tumor marker values and the BCLC stages, the improvement was quite small considering the progress in diagnostic procedures that has been made over the past two decades. The survival of the patients was suboptimal, although it was slightly improved compared with previous reports. The identification of a strategy for identifying individuals with a high risk of developing HCC among patients with non-viral chronic liver diseases is needed to further improve patient survival.

Fatty liver is regarded as the second largest cause of cirrhosis, after chronic viral hepatitis [20, 21]. However, the degree of fat accumulation in the liver is known to decrease with the progression of fatty liver disease [22, 23];
Table 2 Baseline characteristics of patients who practiced moderation in drinking and patients who continued drinking

\begin{tabular}{llll}
\hline & Moderation in drinking & Continuous drinking & $P$ value \\
\hline Number of patients & 311 & 311 & \\
Age, year & $73.0(66.0-78.0)$ & $72.0(65.0-77.0)$ & 0.36 \\
Male gender, $n(\%)$ & $273(87.8)$ & $273(87.8)$ & 1 \\
BMI $\left(\mathrm{kg} / \mathrm{m}^{2}\right)$ & $24.3(22.0-26.7)$ & $23.9(21.6-26.4)$ & 0.64 \\
Alcohol consumption (g/day) & & & 1 \\
$\leq 20, n(\%)$ & $106(34.1)$ & $106(34.1)$ & \\
$21-59, n(\%)$ & $47(15.1)$ & $47(15.1)$ & \\
$\geq 60, n(\%)$ & $158(50.8)$ & $158(50.8)$ & 0.16 \\
Albumin $(\mathrm{g} / \mathrm{dL})$ & $3.7(3.3-4.2)$ & $3.8(3.4-4.2)$ & 0.57 \\
Platelet count $\left(\times 10^{9} / \mathrm{HL}\right)$ & $13.8(9.2-19.4)$ & $14.0(9.7-19.2)$ & \\
\hline
\end{tabular}

$B M I$ body mass index 
Fig. 3 Overall survival of 2011-2015 cohort (solid line) and 1991-2010 cohort (dotted line)

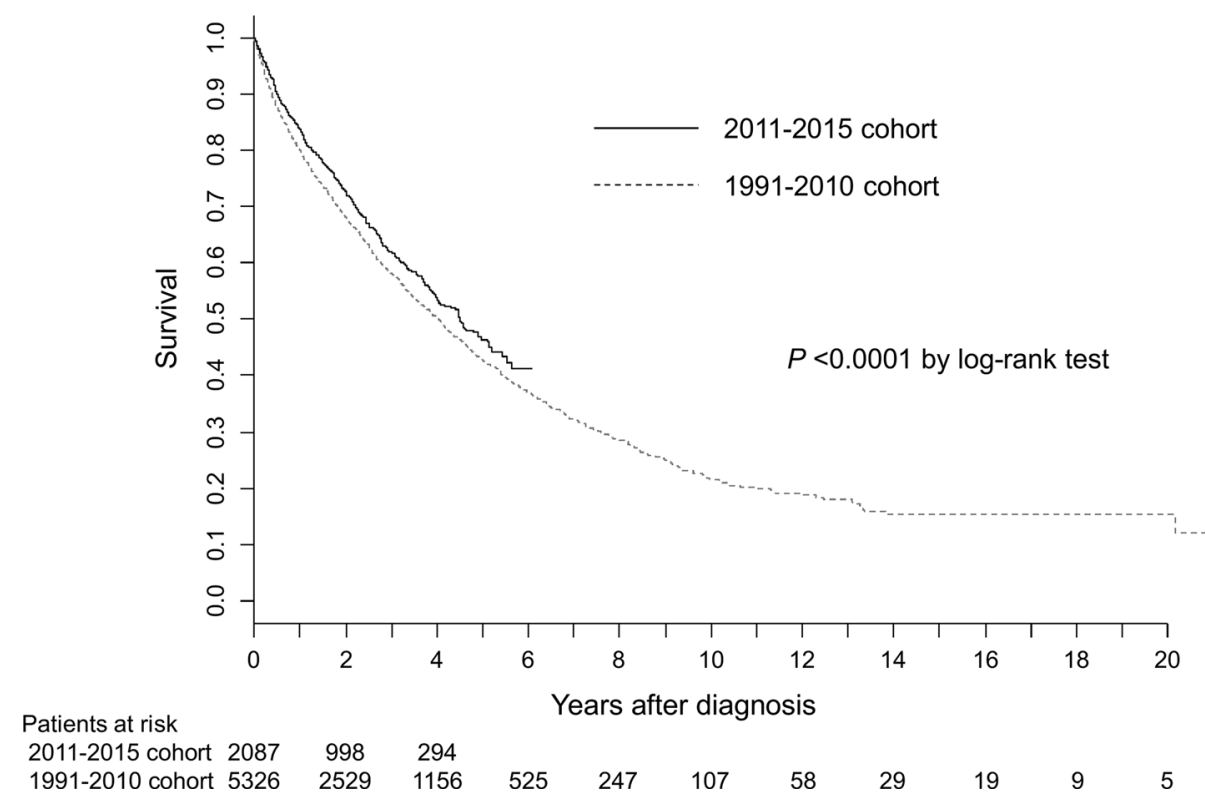

the final stage of this disease, burned-out NASH, is thought to be a major cause of cryptogenic cirrhosis [24, 25]. In fact, we confirmed that at the time of HCC diagnosis, fatty liver had disappeared in $7.1 \%$ of the patients in whom fatty liver had been previously observed. Considering the high prevalences of obesity, diabetes, and hypertension in this cohort, the proportion of patients with a history of fatty liver should be larger than the proportion of patients with fatty liver observed at the time of the diagnosis of HCC.

Among the various mechanisms behind obesity-associated hepatocarcinogenesis, insulin resistance and associated hyperinsulinemia are considered to be key components [26]. Therefore, sulfonylurea, which stimulates beta cells in the pancreas and insulin itself, may increase the risk of $\mathrm{HCC}[27,28]$. On the other hand, metformin reportedly decreases the risk of HCC among diabetic patients [29]. Statins, which are used to treat dyslipidemia (a major component of metabolic syndrome), also reportedly decrease the risk of HCC [30]. Angiotensinconverting enzyme inhibitors and angiotensin II receptor blockers may have a preventive effect on cancer development, including $\operatorname{HCC}[31,32]$. In the current study, we investigated the presence of prescriptions for the treatment of diabetes mellitus, dyslipidemia, and hypertension. Unfortunately, the results were unremarkable; the drug distributions were similar to those in the general population in Japan [33-35]. Clarifying the relationship between prescriptions for the treatment of these diseases and the development of HCC is likely to be difficult, since the popularity and use of different classes of drugs has been changing according to drug development, the accumulation of evidence, and revisions to treatment guidelines.
Although excessive alcohol intake is known to increase the risk of HCC in both viral-related and viral-unrelated chronic liver diseases [36], whether a longer duration of drinking increases the risk remains controversial. Corrao et al. [37] reported that the duration of alcohol intake, in addition to the daily intake, was a risk factor for cirrhosis. On the other hand, Donato et al. [38] reported that while the risk of HCC development increased according to daily alcohol consumption in a linear manner, the duration of drinking and the age at the start of drinking did not affect the risk. They also reported that former drinkers had a higher risk of HCC than current drinkers. Since their study had a case-controlled design, a bias may have existed in that patients who stopped drinking might have lived longer only to eventually develop HCC, while those who continued their excessive drinking might have died from liver failure without having survived long enough to develop HCC. In the current study, we observed an inverse relationship between the duration of drinking and the amount of daily alcohol consumption. These findings suggest that moderate drinking (e.g., daily alcohol intake between 20 and $60 \mathrm{~g}$ ) for longer than 50 years might be a risk factor for HCC. We also showed that the GGT and AST levels decreased significantly as a result of practicing moderation in drinking. Of note, the laboratory data at the time of the diagnosis of HCC might not reflect hidden risks in the past.

Interestingly, the cubic spline hazard according to the BMI exhibited a J-curve similar to that observed in our previous study. In addition, the lowest point was also the same, at around $26 \mathrm{~kg} / \mathrm{m}^{2}$. This finding is somewhat paradoxical, since a BMI $>25 \mathrm{~kg} / \mathrm{m}^{2}$ is reportedly a risk factor for HCC development [39-41]. Regarding this obesity paradox, we previously reported that the skeletal 
Table 3 Multivariate Cox regression analysis of risk factors for overall survival

\begin{tabular}{|c|c|c|}
\hline Variable & HR $(95 \% \mathrm{CI})$ & $P$ value \\
\hline Cohort $2011-2015$ vs. $1991-2010$ & $0.896(0.817-0.983)$ & 0.02 \\
\hline Age per year & $1.02(1.02-1.03)$ & $<0.000$ \\
\hline Female vs. male & $0.816(0.729-0.913)$ & 0.0004 \\
\hline \multicolumn{3}{|l|}{ Alcohol consumption (g/day) } \\
\hline$\leq 20$ & 1 & \\
\hline $21-59$ & $1.02(0.907-1.15)$ & 0.72 \\
\hline$\geq 60$ & $1.03(0.928-1.15)$ & 0.59 \\
\hline \multicolumn{3}{|l|}{ BMI $\left(\mathrm{kg} / \mathrm{m}^{2}\right)$} \\
\hline$<18.5$ & $1.35(0.739-1.13)$ & 0.0009 \\
\hline $18.5-21.9$ & $1.23(1.10-1.37)$ & 0.0003 \\
\hline $22.0-24.9$ & 1 & \\
\hline $25.0-29.9$ & $1.10(0.986-1.22)$ & 0.09 \\
\hline$\geq 30.0$ & $1.23(1.05-1.45)$ & 0.01 \\
\hline \multicolumn{3}{|l|}{ Maximal tumor $(\mathrm{cm})$} \\
\hline$\leq 2.0$ & 1 & \\
\hline $2.1-3.0$ & $1.11(0.973-1.28)$ & 0.12 \\
\hline $3.1-5.0$ & $1.30(1.14-1.49)$ & 0.0001 \\
\hline $5.1-10.0$ & $1.94(1.69-2.22)$ & $<0.0001$ \\
\hline$>10.0$ & $2.97(2.53-3.48)$ & $<0.0001$ \\
\hline \multicolumn{3}{|l|}{ Tumor number } \\
\hline Single & 1 & \\
\hline $2-3$ & $1.39(1.26-1.55)$ & $<0.0001$ \\
\hline $4-5$ & $1.80(1.54-2.11)$ & $<0.0001$ \\
\hline$\geq 6$ & $2.42(2.15-2.74)$ & $<0.0001$ \\
\hline Child-Pugh score (per 1 point) & $1.37(1.34-1.40)$ & $<0.0001$ \\
\hline Extrahepatic metastasis, present & $1.83(1.60-2.09)$ & $<0.0001$ \\
\hline Vascular invasion, present & $1.57(1.27-1.95)$ & $<0.0001$ \\
\hline \multicolumn{3}{|l|}{ AFP (ng/mL) } \\
\hline$<20$ & 1 & \\
\hline 20-99 & $1.47(1.30-1.66)$ & $<0.0001$ \\
\hline 100-399 & $1.97(1.70-2.28)$ & $<0.0001$ \\
\hline$\geq 400$ & $2.22(1.99-2.48)$ & $<0.0001$ \\
\hline
\end{tabular}

$A F P$ alpha-fetoprotein

muscle mass significantly affects the survival of HCC patients [42]. Patients with sarcopenia, a condition in which the skeletal muscle mass is depleted, might have been included among the underweight population, which would have reduced the overall survival rate in this group.

The increase in the BMI in the present study was relatively small, whereas the increase in the proportion of patients with obesity-related disorders was more prominent. This is probably because the median age of the present cohort was 3 years older than that in the previous study, and BMI decreases in accordance with age, reflecting reductions in muscle mass.

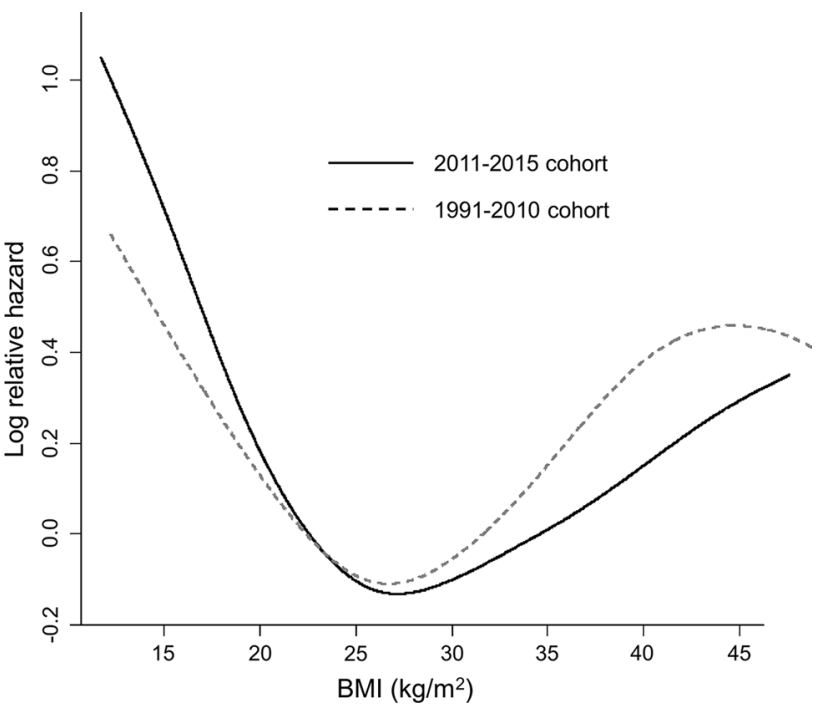

Fig. 4 Estimated hazard function of BMI. The relative hazard was lowest at a BMI of $\sim 26 \mathrm{~kg} / \mathrm{m}^{2}$ for both the 2011-2015 cohort (solid line) and the 1991-2010 cohort (dotted line)

The present study had several limitations. First, as this study did not have a control arm for patients with viral hepatitis, we cannot estimate the impact of lifestyle-related risk factors. Second, since this study was an observational study, we cannot prove the causal relationship between the increased proportion of non-viral HCC and the increasing prevalence of obesity in Japan. However, considering the fact that alcohol consumption per capita has not increased for the last 2 decades [43] and the number of patients with diabetes is increasing in Japan [44], it is highly likely that the increasing prevalence of obesity among Japanese males has contributed to an increase in non-viral HCC.

In conclusion, the incidence of non-B, non-C HCC continued to increase during the current study period of 2011-2015. A strategy for early diagnosis needs to be established to improve patient survival. Moderate drinking for a long duration might be a risk factor for HCC. The study also reconfirmed that patients who were slightly overweight lived the longest.

Acknowledgements This research was supported by AMED under Grant Number JP17fk0210304 and JP18fk0210040 and the Health, Labour and Welfare Policy Research Grants from the Ministry of Health, Labour, and Welfare of Japan (Policy Research for Hepatitis Measures [H30-Kansei-Shitei-003]).

The following investigators enrolled patients in the Inuyama NOBLESSE Study: Hiroshi Aikata (Hiroshima University, Hiroshima); Kyoko Oura (Kagawa University, Takamatsu); Masaaki Kitahara (Kanazawa University, Kanazawa); Motohiko Tanaka (Kumamoto University, Kumamoto); Kenji Oyama (Tottori University, Yonago); Naota Taura (Nagasaki University, Nagasaki); Hiroko Ito (Yokohama City University Medical Center, Yokohama); Akinobu Tawada (Chiba University, Chiba); Shinichiro Nakamura (Okayama University, Okayama); Issei Saeki (Yamaguchi University, Ube); Yasuo Arakawa (Nihon University); Takumi Kawaguchi (Kurume 
University, Kurume); Koji Takai (Gifu University, Gifu); Shigemune Bekki (National Hospital Organization Nagasaki Medical Center, Nagasaki); Naotaka Shimozato (Nara Medical University, Kashihara); Koichi Honda (Oita University, Yufu); Toshihide Shima (Saiseikai Suita Hospital, Suita); Ryotaro Sakamori (Osaka University, Suita); Hiroki Nishikawa (Hyogo College of Medicine, Nishinomiya); Masatoshi Kudo, Naoshi Nishida (Kindai University, Sayama); Masahiro Arai, Toru Arano (Toshiba Hospital, Tokyo); Akira Kato (Shimonoseki Medical Center, Shimonoseki); Akio Ido, Tsutomu Tamai (Kagoshima University, Kagoshima); Yoichi Hiasa, Masashi Hirooka (Ehime University, Toon); Sumio Watanabe, Kazuyoshi Kon (Juntendo University, Tokyo); Shigetoshi Fujiyama, Shiho Miyase (Kumamoto Shinto General Hospital, Kumamoto); Hideyuki Nomura, Nobuyuki Yamashita (Shin-Kokura Hospital, Kitakyushu); Yasuhito Tanaka, Etsuko Iio (Nagoya City University, Nagoya); Keisuke Hino, Yasuyuki Tomiyama (Kawasaki Medical School, Kurashiki).

Open Access This article is distributed under the terms of the Creative Commons Attribution 4.0 International License (http://crea tivecommons.org/licenses/by/4.0/), which permits unrestricted use, distribution, and reproduction in any medium, provided you give appropriate credit to the original author(s) and the source, provide a link to the Creative Commons license, and indicate if changes were made.

\section{References}

1. de Martel C, Maucort-Boulch D, Plummer M, et al. World-wide relative contribution of hepatitis $\mathrm{B}$ and $\mathrm{C}$ viruses in hepatocellular carcinoma. Hepatology. 2015;62:1190-200.

2. Bertuccio P, Turati F, Carioli G, et al. Global trends and predictions in hepatocellular carcinoma mortality. J Hepatol. 2017;67:302-9.

3. Ryerson AB, Eheman CR, Altekruse SF, et al. Annual Report to the Nation on the Status of Cancer, 1975-2012, featuring the increasing incidence of liver cancer. Cancer. 2016;122:1312-37.

4. Jee SH, Sull JW, Park J, et al. Body-mass index and mortality in Korean men and women. N Engl J Med. 2006;355:779-87.

5. Calle EE, Rodriguez C, Walker-Thurmond K, et al. Overweight, obesity, and, mortality from cancer in a prospectively studied cohort of US adults. N Engl J Med. 2003;348:1625-38.

6. Collaboration Emerging Risk Factors. Diabetes mellitus, fasting glucose, and risk of cause-specific death. $\mathrm{N}$ Engl J Med. 2011;364:829-41.

7. Bhaskaran K, Douglas I, Forbes H, et al. Body-mass index and risk of 22 specific cancers: a population-based cohort study of 5.24 million UK adults. Lancet. 2014;384:755-65.

8. Giovannucci E, Harlan DM, Archer MC, et al. Diabetes and cancer: a consensus report. CA Cancer J Clin. 2010;60:207-21.

9. El-Serag HB, Hampel H, Javadi F. The association between diabetes and hepatocellular carcinoma: a systematic review of epidemiologic evidence. Clin Gastroenterol Hepatol. 2006;4:369-80.

10. El-Serag HB, Rudolph KL. Hepatocellular carcinoma: epidemiology and molecular carcinogenesis. Gastroenterology. 2007;132:2557-76.

11. Islami F, Miller KD, Siegel RL, et al. Disparities in liver cancer occurrence in the United States by race/ethnicity and state. CA Cancer J Clin. 2017;67:273-89.

12. Tateishi R, Okanoue T, Fujiwara N, et al. Clinical characteristics, treatment, and prognosis of non- $\mathrm{B}$, non- $\mathrm{C}$ hepatocellular carcinoma: a large retrospective multicenter cohort study. J Gastroenterol. 2015;50:350-60.
13. The committee for revision of the Clinical Practice Guidelines for Hepatocellular Carcinoma. Clinical practice guidelines for hepatocellular carcinoma-the Japan society of hepatology 2009 update. Hepatol Res. 2010;40:16-47.

14. Pugh RN, Murray-Lyon IM, Dawson JL, et al. Transection of the oesophagus for bleeding oesophageal varices. $\mathrm{Br} \mathrm{J}$ Surg. 1973;60:646-9.

15. Sterling RK, Lissen E, Clumeck N, et al. Development of a simple noninvasive index to predict significant fibrosis in patients with HIV/HCV coinfection. Hepatology. 2006;43:1317-25.

16. European Association for the Study of the Liver. EASL clinical practice guidelines: management of hepatocellular carcinoma. J Hepatol. 2018;69:182-236.

17. Japanese Society for Biomedical Research on Alcohol. Japanese Society for Biomedical Research on Alcohol Diagnostic Criteria for Alcoholic Liver Disease 2011 Edition. Available at: http:// www.kanen.ncgm.go.jp/cont/010/sankou.html. Accessed 20 Oct 2018.

18. Liver Cancer Study Group of Japan. General rules for the clinical and pathological study of primary liver cancer, second. English ed. Tokyo: Kasahara; 2003.

19. National Cancer Center, Cancer Information Service. Cancer incidence and incidence rates in Japan in 2014. 2014. Available at: https://ganjoho.jp/reg_stat/statistics/stat/summary.html. Accessed 20 Oct 2018.

20. Wong RJ, Aguilar M, Cheung R, et al. Nonalcoholic steatohepatitis is the second leading etiology of liver disease among adults awaiting liver transplantation in the United States. Gastroenterology. 2015;148:547-55.

21. Sherif ZA, Saeed A, Ghavimi S, et al. Global epidemiology of nonalcoholic fatty liver disease and perspectives on US minority populations. Dig Dis Sci. 2016;61:1214-25.

22. Powell EE, Cooksley WG, Hanson R, et al. The natural history of nonalcoholic steatohepatitis: a follow-up study of forty-two patients for up to 21 years. Hepatology. 1990;11:74-80.

23. Abdelmalek M, Ludwig J, Lindor KD. Two cases from the spectrum of nonalcoholic steatohepatitis. J Clin Gastroenterol. 1995;20:127-30.

24. Ong J, Younossi ZM, Reddy V, et al. Cryptogenic cirrhosis and posttransplantation nonalcoholic fatty liver disease. Liver Transpl. 2001;7:797-801.

25. Caldwell SH, Oelsner DH, Iezzoni JC, et al. Cryptogenic cirrhosis: clinical characterization and risk factors for underlying disease. Hepatology. 1999;29:664-9.

26. Karagozian R, Derdak Z, Baffy G. Obesity-associated mechanisms of hepatocarcinogenesis. Metabolism. 2014;63:607-17.

27. Donadon V, Balbi M, Ghersetti M, et al. Antidiabetic therapy and increased risk of hepatocellular carcinoma in chronic liver disease. World J Gastroenterol. 2009;15:2506-11.

28. Monami M, Lamanna C, Balzi D, et al. Sulphonylureas and cancer: a case-control study. Acta Diabetol. 2009;46:279-84.

29. Decensi A, Puntoni M, Goodwin P, et al. Metformin and cancer risk in diabetic patients: a systematic review and meta-analysis. Cancer Prev Res (Phila). 2010;3:1451-61.

30. Singh S, Singh PP, Singh AG, et al. Statins are associated with a reduced risk of hepatocellular cancer: a systematic review and meta-analysis. Gastroenterology. 2013;144:323-32.

31. George AJ, Thomas WG, Hannan RD. The renin-angiotensin system and cancer: old dog, new tricks. Nat Rev Cancer. 2010;10:745-59.

32. Ho CM, Lee $\mathrm{CH}$, Lee MC, et al. Comparative effectiveness of angiotensin-converting enzyme inhibitors and angiotensin II receptor blockers in chemoprevention of hepatocellular carcinoma: a nationwide high-risk cohort study. BMC Cancer. 2018;18:401. 
33. Oishi M, Yamazaki K, Okuguchi F, et al. Changes in oral antidiabetic prescriptions and improved glycemic control during the years 2002-2011 in Japan (JDDM32). J Diabetes Investig. 2014;5:581-7.

34. Ministry of Health Labour and Welfare of Japan. NDB Open data. 2016; Available from: https://www.mhlw.go.jp/stf/seisaku nitsuite/bunya/0000177182.html. Accessed 20 Oct 2018.

35. Kohro T, Yamazaki T, Sato H, et al. The impact of a change in hypertension management guidelines on diuretic use in Japan: trends in antihypertensive drug prescriptions from 2005 to 2011. Hypertens Res. 2013;36:559-63.

36. Morgan TR, Mandayam S, Jamal MM. Alcohol and hepatocellular carcinoma. Gastroenterology. 2004;127:S87-96.

37. Corrao G, Arico S, Lepore R, et al. Amount and duration of alcohol intake as risk factors of symptomatic liver cirrhosis: a case-control study. J Clin Epidemiol. 1993;46:601-7.

38. Donato F, Tagger A, Gelatti U, et al. Alcohol and hepatocellular carcinoma: the effect of lifetime intake and hepatitis virus infections in men and women. Am $\mathrm{J}$ Epidemiol. 2002;155:323-31.
39. Ioannou GN, Splan MF, Weiss NS, et al. Incidence and predictors of hepatocellular carcinoma in patients with cirrhosis. Clin Gastroenterol Hepatol. 2007;5:938-45.

40. Ohki T, Tateishi R, Sato T, et al. Obesity is an independent risk factor for hepatocellular carcinoma development in chronic hepatitis C patients. Clin Gastroenterol Hepatol. 2008;6:459-64.

41. Yu MW, Shih WL, Lin CL, et al. Body-mass index and progression of hepatitis B: a population-based cohort study in men. J Clin Oncol. 2008;26:5576-82.

42. Fujiwara N, Nakagawa H, Kudo Y, et al. Sarcopenia, intramuscular fat deposition, and visceral adiposity independently predict the outcomes of hepatocellular carcinoma. J Hepatol. 2015;63:131-40.

43. World Health Organization. Global status report on alcohol and health. 2014. Available at: http://www.who.int/substance_abuse/ activities/gsrah/en. Accessed 20 Oct 2018.

44. Ministry of Health Labour and Welfare of Japan. National Health and Nutrition Examination Survey. 2017. Available at: https:// www.mhlw.go.jp/stf/houdou/0000177189_00001.html. Accessed 20 Oct 2018. 